\title{
OPERATIONAL EXPERIENCE WITH THE PEP-II TRANSVERSE COUPLED-BUNCH FEEDBACK SYSTEMS*
}

\author{
W. Barry, J. Byrd, J. N. Corlett, D. Li, LBNL Center for Beam Physics, Berkeley, CA, \\ J. Fox, M. Minty, S. Prabhaker, D. Teytelman, SLAC, Stanford, CA.
}

\begin{abstract}
Operational experience with the PEP-II high energy ring (HER) and low energy ring (LER) transverse coupled-bunch feedback systems is discussed. In particular, some key performance data including beam transfer function, mode spectrum, and some growth rate observations are presented. In general, growth rates much greater than expected have been observed in the HER. LER growth rates have not been measured but are thought to be lower than those of the HER based on lower feedback gains required for LER beam stabilization. Some results from experiments using the longitudinal feedback system electronics in conjunction with the transverse feedback system to study the HER instabilities are presented.
\end{abstract}

\section{INTRODUCTION}

The PEP-II B-Factory [1] is a high-luminosity, asymmetric electron-positron collider consisting of a 9 $\mathrm{GeV}, 1.0$ A high-energy electron storage ring (HER), and a $3.1 \mathrm{GeV}, 2.14$ A low-energy positron storage ring (LER). Because of the high average beam currents in both rings, active feedback systems [2] are used to suppress the growth of transverse coupled-bunch instabilities.

During the past year, PEP-II has completed several very successful commissioning periods, the most recent being the Jan/Feb 1999 run during which both rings achieved substantial currents ( $>500 \mathrm{~mA}$ HER and $>1100$ $\mathrm{mA}$ LER) and significant luminosity $\left(5 \times 10^{32}\right)$ was obtained [3]. At these high currents, the transverse feedback systems played a critical role in beam containment and stabilization.

In general, the HER has been observed to be less stable than the LER. Typical HER transverse instability thresholds are on the order of ten milliamps while LER thresholds are in the $100 \mathrm{~mA}$ area. Feedback gains required to stabilize the two beams roughly reflect the order of magnitude difference in thresholds. Growth rates four-to-ten times greater than expected for the HER have been measured [2]. LER growth rates have not been measured but will be during the next run. Experience thus far with the LER points towards transverse growth rates more in line with predicted rates (table 1). Many

\footnotetext{
*Supported by the US Department of Energy under contract numbers DE-AC03-76SF00098 (LBNL) and AC03-76SF00515 (SLAC).
}

experiments and a great deal of data has been taken to try to determine the origin of the HER instabilities. Work towards a conclusion in this area is ongoing and will be published at a future date.

\section{PARAMETER AND SYSTEM REVIEW}

A brief list of accelerator and transverse feedback system design parameters appears in Table 1. Nominally, PEP-II operates with every other bucket filled $(238 \mathrm{MHz}$ bunch rate). This sets the minimum bandwidth for the feedback system at $119 \mathrm{MHz}$. However, the electronics has been designed to have a bandwidth of $250 \mathrm{MHz}$ to allow for possible operation with every bucket filled. The kickers cover DC-119 $\mathrm{MHz}$ for maximum shunt impedance in the every-other-bucket operating mode. They can be replaced with $238 \mathrm{MHz}$ versions if an everybucket fill becomes a likely operating mode. The feedback systems are designed to provide a damping rate that is approximately three times greater than the growth rate of the fastest expected (vertical resistive wall) coupled-bunch mode.

Table 1: Accelerator / feedback design parameters.

\begin{tabular}{ccc}
\hline \hline Parameter & Description & HER / LER Value \\
\hline $\mathrm{E}$ & Beam energy & $9.0 / 3.1 \mathrm{GeV}$ \\
$\mathrm{f}_{\mathrm{rf}}$ & RF frequency & $476 \mathrm{MHz}$ \\
- & Bucket space & $2.1 \mathrm{~ns}$ \\
- & Bunch space & $4.2 \mathrm{~ns}$ \\
$\mathrm{I}_{\mathrm{av}}$ & Average current & $1.0 / 2.14 \mathrm{~A}$ \\
$\mathrm{f}_{0}$ & Orbit frequency & $136.3 \mathrm{kHz}$ \\
$v_{\mathrm{V}}$ & Vertical tune & $23.64 / 34.64$ \\
$v_{\mathrm{h}}$ & Horizontal tune & $24.57 / 36.57$ \\
$\alpha_{\mathrm{V}}$ & Vertical R-wall & $0.26 / 1.09 \mathrm{~ms}^{-1}$ \\
& growth rate (calc) & \\
$\alpha_{\mathrm{h}}$ & Horizontal R-wall & $0.18 / 0.71 \mathrm{~ms}^{-1}$ \\
& growth rate (calc) & \\
$\alpha_{\mathrm{f}}$ & Feedback design & $3.2 \mathrm{~ms}^{-1}$ \\
& damping rate & \\
Required feedback bandpass & $13.6 \mathrm{kHz}-119 \mathrm{MHz}$ \\
Feedback electronic bandpass & $10 \mathrm{kHz}-250 \mathrm{MHz}$ \\
Kicker bandpass & DC - 119 MHz \\
\hline \hline
\end{tabular}

The feedback system diagram is shown in figure 1 . Beam moment signals $(\mathrm{I} \Delta \mathrm{x})$ from two sets of pickups are detected with microwave receivers at $3 \mathrm{f}_{\mathrm{rf}}$. After downconversion to baseband, the signals are proportionately summed to produce a correction signal that is 90 degrees 
out of phase with beam position at the kickers. A digital delay provides the pickup-to-kicker timing and the kicker electrodes are individually driven differentially with 120 W class-A power amplifiers. Other system features include a provision for single-bunch kickout, predigitization orbit-offset-rejection electronics, and fast switches to gate the feedback on/off for grow damp measurements with the longitudinal feedback system front-end.

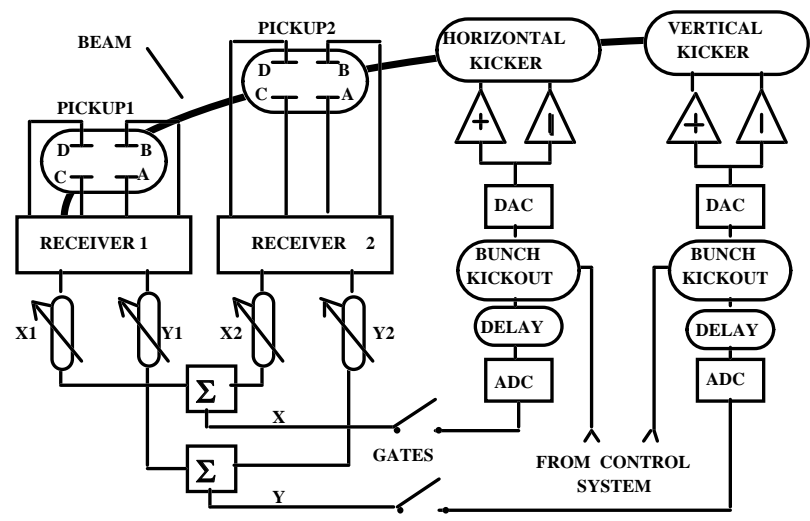

Figure 1: Transverse feedback system.

\section{LER OPERATION}

Commissioning of the LER began in July, 1998. Experience gained with the HER feedback system made the LER system commissioning a simple and straight forward task. The system was quickly timed using the simple technique described in reference [2]. For rough phasing, the signs of the signals from each of the two pickups were simply set for stability without measuring transfer phase. The system performed well in this rough configuration and was subsequently left alone to make way for other commissioning tasks.

At the beginning of the Jan/Feb 99 run, the LER system was properly phased using a network analyzer in anticipation of going to higher currents. An example vertical transfer function for the properly tuned system appears in figure 2. In this configuration, the system stabilized the beam to the highest currents to date, $>1100$ $\mathrm{mA}$.

Because of the early success, only limited time was allotted to LER coupled-bunch mode studies. Despite the limited experimental time, some important stability observations were made for the LER. In particular, the beam was found to be less stable in the horizontal plane. This is contrary to theory which indicates that the strongest expected source of instability is the vertical resistive wall impedance. The threshold for horizontal instability is in the $100 \mathrm{~mA}$ region. The vertical threshold was not measured but appears to be much higher. The effect of horizontal feedback on the LER beam at $100 \mathrm{~mA}$ is shown over the first ten orbit harmonics in figures 3 and
4. In general, the strongest unstable modes appeared at frequencies below 3 or $4 \mathrm{MHz}$ indicating horizontal resistive wall as a possible source. More detailed measurements for the LER including modal growth rates are planned for the next run beginning May 99.
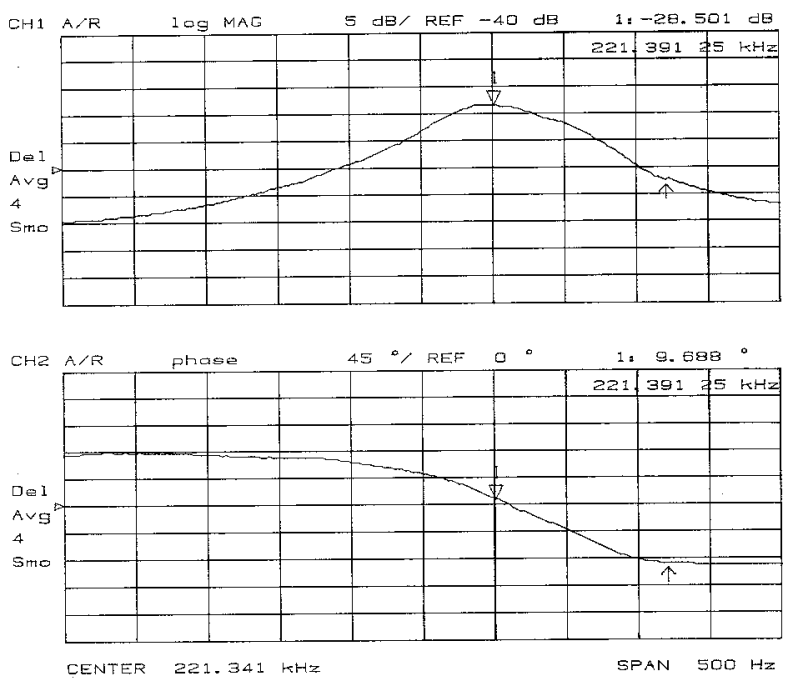

Figure 2: Transfer function of lower vertical sideband of second orbit harmonic (LER).

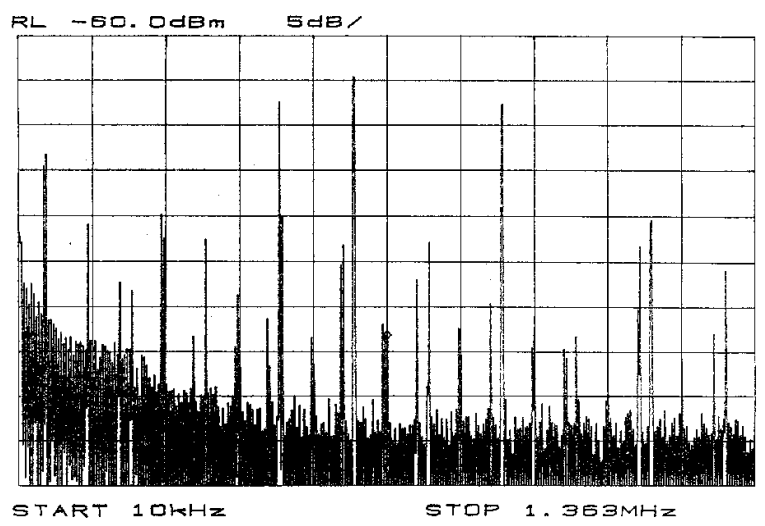

Figure 3: Spectrum of horizontal sidebands about first ten orbit harmonics, feedback off (LER, $100 \mathrm{~mA}$ ).

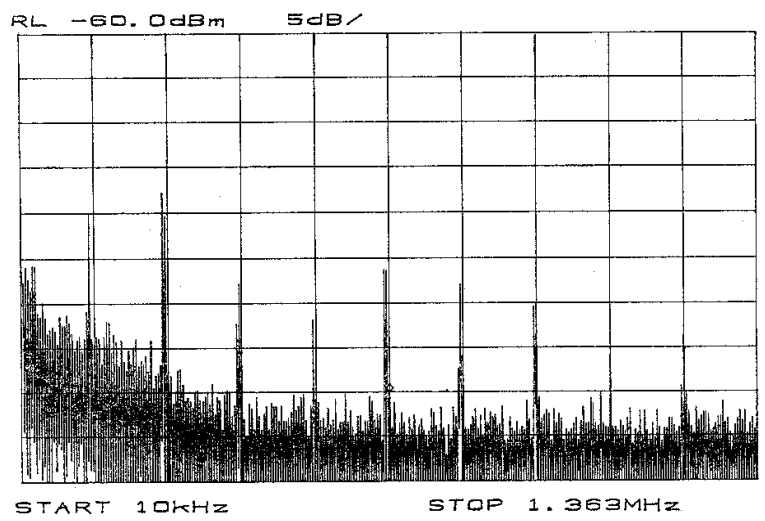

Figure 4: LER spectrum about first ten orbit harmonics with horizontal feedback on, 100 ma. 


\section{HER OPERATION}

During the past several commissioning periods, efforts have been focused on finding the source of the fast HER instabilities $[4,5]$. Some brief comments on measurement techniques and initial observations are discussed here.

The measurement technique uses one of the longitudinal feedback systems to gate the transverse feedback system off and on with broadband GaAs FET Tsection switches allowing beam motion to grow then damp. Bunch-by-bunch transverse motion from the transverse receivers during the grow/damp is recorded and processed with the longitudinal feedback system front-end and DSP farm. With off-line analysis, modal growth/damping rates, closed-loop feedback system behavior, and beam impedance information can be obtained.

An example measurement using this technique for the horizontal plane of the HER is shown in figures 5 and 6 for a 290 bunch, $40 \mathrm{~mA}$ beam. Figure 5 shows bunch-bybunch growth and damping as a function of time. Using Fourier transform techniques, the modal structure of the beam vs. time can be obtained as shown in figure 6 . In this case, two low-frequency modes are clearly present. Also note that the growth is non-exponential and that for one mode, the beam decays with feedback to a steady state level. Although not measured here, the growth rates are known to be large and amplitude dependent with the fastest rates occurring for small amplitudes. Thus one explanation for the residual level is that the mode grows to an amplitude (with decreasing growth rate) to where it is marginally controlled by the feedback system gain.

One consequence of extremely high growth rates is that correspondingly high feedback gains are required to stabilize the beam. At these high gains, the system is extremely sensitive to residual orbit-offset signal saturation effects. Efforts to reduce residual orbit-offset signal levels even further are presently underway.

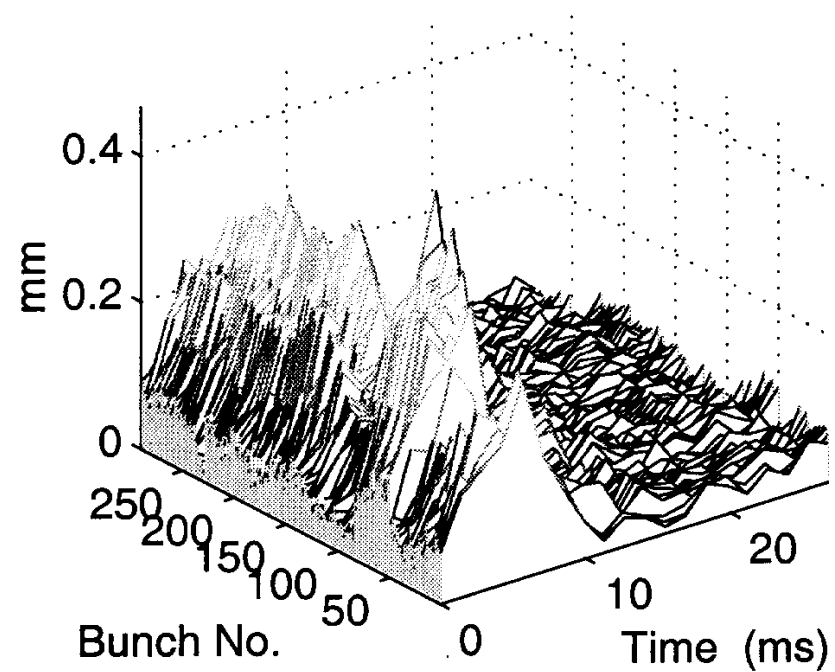

Figure 5: HER horizontal time domain bunch-by-bunch oscillation envelopes, $\mathrm{I}_{\mathrm{av}}=40 \mathrm{~mA}, 290$ bunches.

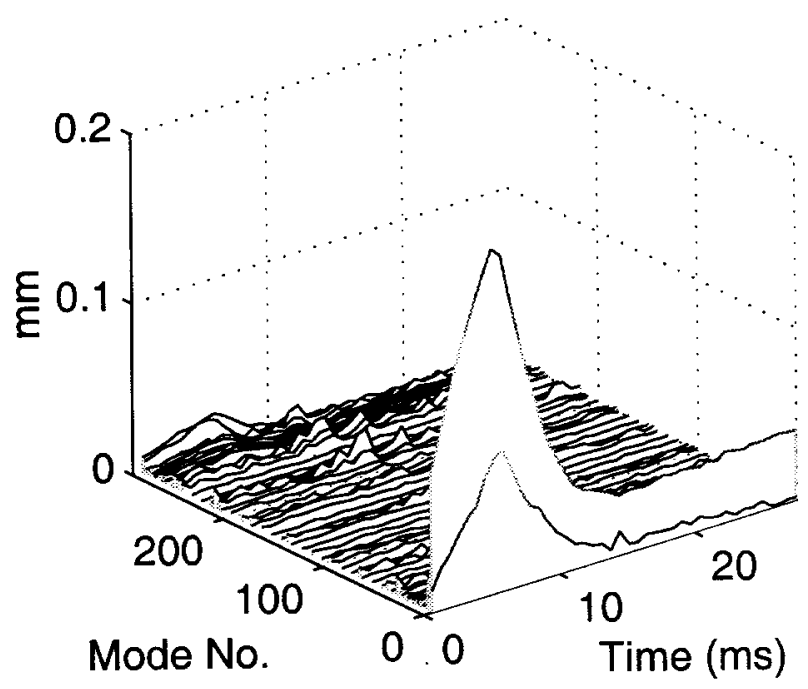

Figure 6: HER time evolution of horizontal modes, $\mathrm{I}_{\mathrm{av}}=40 \mathrm{~mA}, 290$ bunches.

\section{CONCLUSION}

Both the HER and LER transverse coupled-bunch feedback systems are operational and in use for controlling instabilities in both rings. Observations thus far indicate that the LER is generally well behaved with unstable modes easily controlled by the feedback system. Instabilities in the HER, although basically controlled by the feedback system, are more severe with growth rates much greater than expected. During the next run, HER stability studies will continue, growth rates in the LER will be measured, orbit offset rejection will be improved, and the single-bunch kickout feature for both systems will be tested.

\section{REFERENCES}

[1] "PEP-II Conceptual Design Report", LBL-PUB5379, SLAC-418, CALT-68-1869, UCRL-ID114055, UC-IIRPA-93-01, June, 1993.

[2] W. Barry, et al, "Initial Commissioning Results From the PEP-II Transverse Coupled-Bunch Feedback Systems", Proceedings of the 1998 European Particle Accelerator Conference, Stockholm, Sweden, June 1998.

[3] J. Seeman, "Commissioning Results of B-Factories", These Proceedings

[4] D. Teytelman, et al, "Transverse Multibunch Instability Diagnostics via Downsampled Transient Techniques", These Proceedings.

[5] S. Prabhaker, et al, "Measurement of a Fast Vertical Instability in the PEP-II HER", SLAC PEP-II-APNote-99-04, 1998. 